\title{
Características cinéticas durante a marcha de um músico com e sem o transporte de seu instrumento
}

\author{
Kinetic characteristics of the gait of a musician \\ carrying or not his instrument
}

\author{
Clarissa Stefani Teixeira ${ }^{1}$ \\ Fausto Kothe 2 \\ Érico Felden Pereira ${ }^{3}$ \\ Carlos Bolli Mota ${ }^{4}$
}

1 Universidade Federal de Santa Catarina. Programa de Pós-Graduação em Engenharia de Produção - Ergonomia. Florianópolis, SC. Brasil.

2 Universidade Federal de Santa Maria. Curso de Música. Santa Maria, RS. Brasil.

3 Universidade Federal do Paraná. Programa de PósGraduação em Educação Física - Cronobiologia. Curitiba, PR. Brasil.

4 Universidade Federal de Santa Maria. Laboratório de Biomecânica. Santa Maria, RS. Brasil.

Recebido em 07/01/08 Aprovado em 28/07/08
Resumo - A integridade do sistema locomotor pode ser comprometida pelo transporte de determinados objetos, principalmente, quando esse é realizado de uma forma inadequada. $\mathrm{O}$ transporte de instrumentos musicais, devido ao seu peso e tamanho, pode contribuir para o surgimento de disfunções corporais nos músicos que necessitam realizá-lo com freqüência, influenciando no seu equilíbrio e postura corporal. Logo, buscou-se investigar qual a força de reação do solo durante a marcha de um músico com e sem o transporte de seu instrumento. Para a aquisição dos dados cinéticos, foram utilizadas duas plataformas de força AMTI (Advanced Mechanical Technologies, Inc.). O sujeito totalizou 40 tentativas, sendo 20 sem o transporte do instrumento e 20 com o transporte do instrumento. Todas as variáveis analisadas, através do teste $t$, apresentaram diferenças estatisticamente significativas com e sem o transporte do instrumento. Com isso, pode-se perceber que ao se transportar alguma carga, como nesse caso de 7,75\% de seu próprio peso, o aparelho locomotor sofre alterações.

Palavras-chave: Marcha; Equilíbrio; Saúde do trabalhador.

Abstract - The integrity of the locomotor system can be compromised by the transport of certain objects, especially when done in an inadequate manner. Due to their weight and size, the transport of musical instruments can contribute to body dysfunctions in musicians who frequently have to carry their instruments, influencing balance and body posture. Thus, the soil reaction force was investigated during the gait of a musician carrying or not his instrument. Two AMTI (Advanced Mechanical Technologies, Inc.) platforms were used for kinetic data acquisition. A total of 40 measurements were obtained for gait and balance: 20 without carrying the instrument and 20 while carrying the instrument. The $t$ test showed significant differences between the two situations for all variables analyzed. The results suggest that the locomotor system suffers alterations when carrying any kind of load, as was the case here in which the subject carried $7.75 \%$ of his own weight.

Key words: Gait; Balance; Occupational health. 


\section{INTRODUÇÃO}

Do período de formação ao ingresso no mercado de trabalho, os músicos são confrontados a um alto estresse ocupacional que assume manifestações diversas como, por exemplo, incidentes musculares ocasionados pelo uso excessivo da musculatura envolvida no tocar, pela repetição de movimentos e pela manutenção de posturas fixas em um longo período ${ }^{1-4}$. Exames ortopédicos, posturais e cinesiológicos através da observação dos músicos em ação possibilitaram detectar má postura, relacionada ao instrumento ou não, inadequações posturais devido a vícios técnicos, inadequação dos acessórios, problemas técnicos causadores de contratura muscular ou tensão excessiva articular ou neuromuscular e a presença de doenças orgânicas de natureza articular ou periarticular ${ }^{5-7}$.

A observação da postura adotada pelo instrumentista pode ser esclarecedora à medida que ela evidencia os desequilíbrios adquiridos. Ombros acentuadamente assimétricos, lateralidade da pélvis, desenvolvimento dos músculos das mãos e antebraços, escoliose e outros desvios na coluna, cabeça tombada para o lado oposto da movimentação ao instrumento ou estendida à frente são algumas das leituras corporais possíveis para o entendimento das disfunções ${ }^{8}$. Os instrumentos hoje tocados em uma orquestra foram projetados e por vezes, construídos séculos atrás?. Seus princípios permanecem em termos de eficácia acústica, mas o conhecimento disponível sobre a fisiologia humana e biomecânica possibilita questionamentos sobre as mudanças necessárias ao conforto e saúde de quem os toca. A constituição anatômica, a maneira de transportar o instrumento, seu peso e tamanho podem contribuir para o surgimento de disfunções no sistema biológico. Os estojos, construídos para guarda e proteção dos instrumentos, podem interferir no equilíbrio postural do músico em função do seu formato, peso, do tempo e da forma que são por eles carregados. No caso dos violistas, a viola, artefato mediador da atividade do músico violista, quando somada ao estojo, sem acessórios de estudos, pesa aproximadamente $6 \mathrm{kgf}$. Esse peso é transportado pela maioria dos músicos a pé, normalmente, por um percurso que vai de suas residências ou do meio de transporte até o local de trabalho.

Estudos biomecânicos, de acordo com Bertoncello et al. 'indicam duas possibilidades consideradas favoráveis para o transporte de mochilas: 1) a bolsa tipo mochila, transportada na parte frontal do corpo; e, 2) a bolsa bilateral tipo coldre. Porém, sabe-se que ao se transportar o instrumento, os músicos não utilizam nenhum tipo de mochila. Como os estojos disponíveis para a compra normalmente apresentam somente uma alça de agarre, este é carregado, na maioria das vezes, por um braço só, o que possivelmente irá sobrecarregar o hemicorpo desse lado.

Além da marcha ser utilizada na rotina do ser humano, com o passar dos anos ela torna-se deteriorada, podendo, assim, acarretar danos ao sistema locomotor. Um fator importante que pode ser destacado é a força de reação do solo que é originada do contato entre o pé com o solo. Os dados da força de reação do solo têm sido usados em muitos estudos para investigar uma variedade de atividades ${ }^{10-13}$. Para Winter e White ${ }^{14}$, as características envolvidas durante a marcha humana, entre elas a força de reação do solo, têm sido medidas e analisadas mais que qualquer outro padrão singular de movimento. De acordo com Wyss et al..$^{15}$,as técnicas para avaliar os parâmetros da marcha estão disponíveis por, aproximadamente, 150 anos. Hoje, cientistas de muitas universidades, hospitais e laboratórios de biomecânica estão engajados na análise de um grande número de pessoas com marchas normal e patológica ${ }^{16-18}$, mas foram encontrados poucos estudos que tratassem de temas sobre a marcha de indivíduos normais que necessitem carregar seus instrumentos de trabalho durante suas locomoções, como o caso dos músicos. Diante do exposto, buscou-se neste estudo, avaliar variáveis de força de reação vertical do solo de um músico, comparando os resultados intra-membros e com e sem o transporte do instrumento.

\section{PROCEDIMENTOS METODOLÓGICOS}

Foi analisado nesse estudo um sujeito saudável, do sexo masculino, com 26 anos de idade, estatura de 1,83 m e massa corporal de $89 \mathrm{~kg}$, músico profissional, com 9 anos de prática no instrumento viola, considerado ativo fisicamente conforme análise realizada através do Questionário Internacional de Atividade Física ${ }^{19}$.

Para a aquisição dos dados cinéticos, foram utilizadas duas plataformas de força AMTI OR6-6-1000 (Advanced Mechanical Techno- 
logies, Inc.) com as seguintes características de sensibilidade: Força médio-lateral $=0,6632$ $\mu \mathrm{V} / \mathrm{V} / \mathrm{N}$; Força ântero-posterior $=0,6633 \mu \mathrm{V} /$ $\mathrm{V} / \mathrm{N}$; Força vertical $=0,1718 \mu \mathrm{V} / \mathrm{V} / \mathrm{N}$; Momento médio-lateral $=1,6845 \mu \mathrm{V} / \mathrm{V} / \mathrm{Nm}$ ); Momento antero-posterior $=1,6961 \mu \mathrm{V} / \mathrm{V} / \mathrm{Nm}$; Momento vertical $=3,2798 \mu \mathrm{V} / \mathrm{V} / \mathrm{Nm}$.

Para calibração das plataformas, o sujeito posicionou-se com os dois pés sobre a plataforma I e permaneceu na posição anatômica de referência, ou seja, com membros inferiores posicionados na largura do quadril, braços relaxados ao longo do corpo. Para a coleta, foram feitas duas calibrações: a primeira com o sujeito sem a utilização do instrumento e a segunda com o instrumento.

O músico teve um tempo para a ambientação com o laboratório. A coleta desenvolveu-se nas seguintes etapas: 1) denominada situação 1: marcha sem transporte de instrumento; 2) denominada situação 2: marcha com transporte do instrumento. Durante toda a coleta de dados o instrumento foi carregado pelo membro superior direito, pois segundo o músico é nesse lado que ele normalmente o transporta.

As plataformas de força foram posicionadas a cinco milímetros uma da outra. A taxa de amostragem das plataformas foi de $1000 \mathrm{~Hz}$. O sujeito totalizou 40 tentativas para a marcha, sendo 20 sem o transporte do instrumento e 20 com o transporte do instrumento, todas elas sem a utilização de nenhum tipo de calçado, sendo solicitado que realizasse a marcha da mesma forma que faz no seu dia-a-dia. A velocidade da marcha foi controlada por fotocélulas da marca Trony, posicionadas a 6 metros uma da outra. Para a tentativa ser efetivada, a velocidade da marcha foi padronizada entre 4 e $5 \mathrm{~km} / \mathrm{h}$, devendo o músico realizá-la nesse intervalo. Todas as avaliações foram realizadas no Laboratório de Biomecânica da Universidade Federal de Santa Maria.

O sujeito avaliado, após convite e descrição dos procedimentos utilizados no estudo, assinou o Termo de Consentimento Livre e Esclarecido, sendo que o estudo foi protocolado sob número 23081. 010639/2007- 21.

As variáveis estudadas relacionadas à força de reação do solo durante a marcha foram definidas como: força máxima (Fzmáx): corresponde a maior força vertical; tempo da força vertical máxima (tFzmáx): corresponde ao tempo em que ocorreu a força vertical máxima; e impulso (Imp): corresponde à área sob a curva de força vertical, e estão ilustradas na Figura 1. Foi solicitado que para a execução da marcha o sujeito utilizasse uma velocidade confortável.
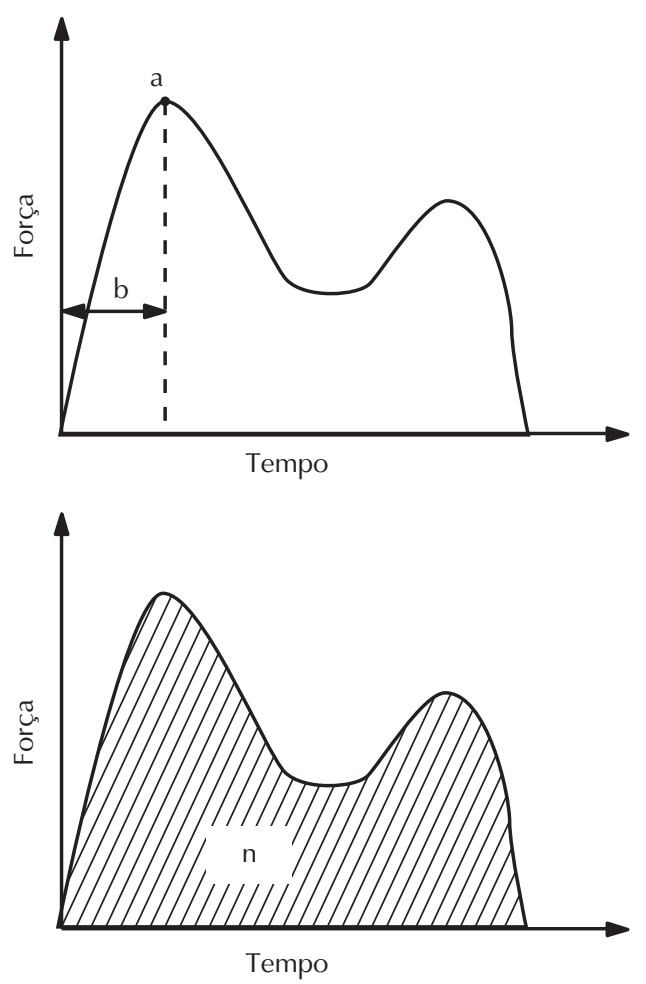

Figura 1. Variáveis da componente de força vertical: primeiro pico de força vertical (a), tempo do primeiro pico de força (b), e impulso (n).

A força vertical máxima e o impulso foram normalizados pelo peso corporal (PC) do sujeito e o tempo da força vertical máxima foi normalizado pelo tempo total de apoio.

A Figura 2 ilustra o posicionamento das plataformas de força para a coleta de dados da marcha.
Plataforma I

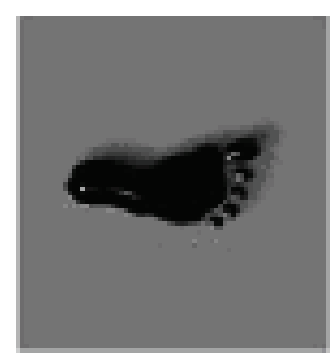

Plataforma II

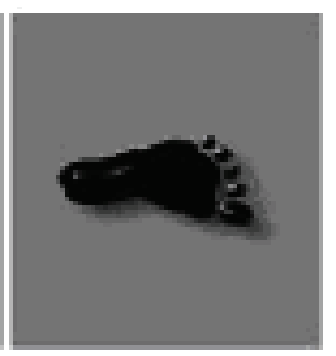

Figura 2. Posicionamento das plataformas para a coleta da marcha.

Os dados foram submetidos a uma estatística descritiva. A normalidade dos dados foi 
Tabela 1. Média, desvio padrão e resultados do teste t de student das variáveis Fzmáx, tFzmáx e Imp para o membro direito e esquerdo sem e com o transporte do instrumento.

\begin{tabular}{|c|c|c|c|c|}
\hline \multirow[b]{2}{*}{ Instrumento } & \multirow[b]{2}{*}{ Variáveis } & Membro direito & Membro esquerdo & \multirow[b]{2}{*}{$\mathrm{t}(\mathrm{p} \text {-valor })^{*}$} \\
\hline & & $\begin{array}{c}\text { Média } \\
\text { (desvio padrão) }\end{array}$ & $\begin{array}{c}\text { Média } \\
\text { (desvio padrão) }\end{array}$ & \\
\hline \multirow{3}{*}{ Sem } & Fzmáx (PC) & $1,10(0,04)$ & $1,08(0,04)$ & $0,99(0,34)$ \\
\hline & tFzmáx (\% do ciclo) & $40,97(29,01)$ & $28,77(17,84)$ & $1,07(0,30)$ \\
\hline & Imp (PC.s) & $0,50(0,02)$ & $0,49(0,01)$ & $1,06(0,31)$ \\
\hline \multirow{3}{*}{ Com } & Fzmáx (PC) & $1,07(0,03)$ & $1,05(0,03)$ & $1,12(0,32)$ \\
\hline & tFzmáx (\% do ciclo) & $65,40(24,00)$ & $49,07(27,59)$ & $1,11(0,21)$ \\
\hline & $\operatorname{Imp}(P C . s)$ & $0,53(0,01)$ & $0,54(0,001)$ & $0,85(0,96)$ \\
\hline
\end{tabular}

* significância do teste t de student

confirmada através do teste de Shapiro-Wilk, o teste $t$ para dados pareados foi aplicado para a comparação entre as médias das variáveis do membro esquerdo e direito, com e sem transporte do instrumento. O nível de significância adotado para todos os testes foi de 5\%.

\section{RESULTADOS}

A seguir serão ilustrados os resultados encontrados durante a execução da marcha pelo músico, com e sem o transporte do seu instrumento. Primeiramente, foram mensuradas a massa da viola e do estojo, que juntos, no momento da coleta, apresentaram 6,9 kg. Avaliando a massa (instrumento e estojo), obteve-se o valor de $7,75 \%$ do peso corporal do sujeito. O sujeito investigado afirmou carregar seu instrumento de 30 a 60 minutos diários.

As Figuras 3 e 4 ilustram o comportamento da força vertical durante uma tentativa da marcha para os membros direito (Figura 3) e esquerdo (Figura 4) com e sem o transporte do instrumento.

membro direito

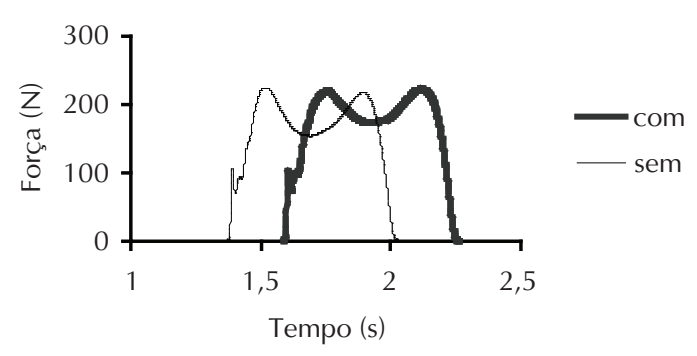

Figura 3. Curvas da força de reação do solo do membro direito com e sem o transporte do instrumento.

A Tabela 1 ilustra a média, o desvio padrão e o nível de probabilidade de significância dos testes de diferenças entre médias das variáveis Fzmáx, tFzmáx e Imp para o membro direito e esquerdo sem e com o transporte do instrumento.

membro esquerdo

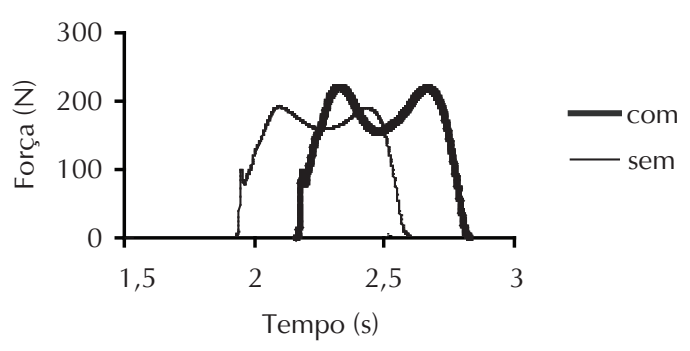

Figura 4. Curvas da força de reação do solo do membro esquerdo com e sem o transporte do instrumento.

Analisando as variáveis referentes à força de reação do solo durante a marcha (Tabela 1), o teste $t$ não mostrou diferença estatisticamente significativa entre nenhuma variável, comparando os membros direito e esquerdo quando o músico realizou a marcha tanto com, quanto sem seu instrumento. Isso remete-nos a inferências sobre a lateralidade dos membros os quais apresentam-se simetricamente durante a marcha, tanto sem quanto com o transporte do instrumento. Com isso os dados passaram a ser analisados juntamente.

Os dados referentes à força vertical máxima durante a marcha nas situações com e sem o transporte do instrumento estão ilustrados na Figura 5. Os valores encontrados nesse estudo para essa força foram de 1,09 \pm 0,04 PC para a situação sem instrumento e de 1,06 \pm 0,03 PC para a situação com instrumento. $O$ teste $t$ aplicado para comparação entre os valores da Fzmáx entre a situação 1 e a situação 2, apresentou diferença estatisticamente significativa nessa variável, com $\mathrm{t}=2,58$ e $\mathrm{p}=0,02$. 


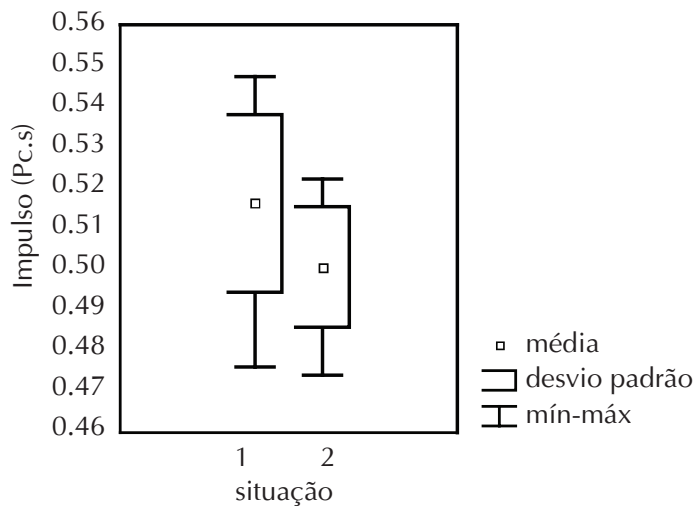

Figura 5. Força vertical máxima durante a marcha de um músico nas situações sem e com o transporte do instrumento.

Quanto ao tempo da força vertical máxima, o teste $t$ mostrou diferenças estatisticamente significativas, sendo encontrados os valores de $34,87 \pm 24,19 \%$ sem o transporte do instrumento e $56,59 \pm 25,85 \%$ com o transporte do instrumento, com probabilidade de significância $\mathrm{p}=0,01$ para um valor de $\mathrm{t}=-2,94$. $\mathrm{O}$ impulso ilustrado na Figura 6, também mostrou diferenças estatisticamente significativas durante a marcha com e sem o transporte do instrumento, com probabilidade de significância $\mathrm{p}<0,001$ para um valor de $\mathrm{t}=-9,7$.

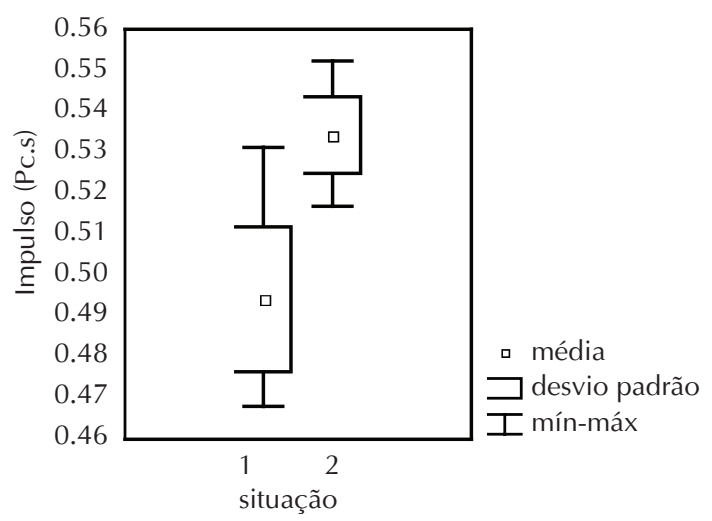

Figura 6. Impulso durante a marcha de um músico sem e com o transporte do instrumento.

\section{DISCUSSÃO}

A preocupação com o trabalho dos indivíduos que transportam cargas, visando o trabalho, não é recente. Page ${ }^{20}$ comparou cinco tipos de mochila: a frontal, a lateral (com e sem cinta) e a transversal (com e sem cinta). Através de modelagem e análise biomecânica das forças de contração dos músculos do tronco e das cargas compressoras da coluna vertebral, resultantes da carga externa das mochilas, procurou-se conhecer as forças compressoras que agem sobre o disco L3 e a força muscular de 22 músculos das costas, objetivando discutir o balanceamento dessas forças na coluna e a relação do peso assimétrico com a fadiga muscular. Também realizou análise eletromiográfica das atividades do músculo em três diferentes níveis do dorso, visando a validação da análise biomecânica. $\mathrm{O}$ experimento foi realizado com postura estática e dinâmica, com carga total (mochila e conteúdo). O autor concluiu que os picos de forças compressoras no uso das mochilas lateral e transversal foi bastante alto; a mochila lateral com cinta teve um resultado melhor; as mochilas laterais e transversais apresentam importantes diferenças relativas entre a força muscular nos lados direito e esquerdo. Os mesmos autores ainda concluem que a mochila frontal requer uma porcentagem menor de força voluntária máxima dos sujeitos. No caso do presente estudo, estas afirmações tornam-se pertinentes, pois o instrumento é carregado durante a marcha somente em um lado do corpo, e como afirma o sujeito, na maior parte do tempo em seu lado direito.

Ren et al. ${ }^{21}$ demonstram que a marcha, por períodos de apenas 5 minutos, pode levar à fadiga ou ao alongamento adaptativo da musculatura, provocando, assim, alterações posturais. Este fato torna-se preocupante, pois os músicos, tanto aqueles que utilizam-o instrumento viola, quanto aqueles que utilizam outros instrumentos necessitam da boa integridade física da musculatura para a execução dos movimentos específicos de cada instrumento. Deve-se aqui, ressaltar que, dos instrumentos de cordas o instrumento viola pode ser considerado o segundo menor instrumento, sendo que a variação do tamanho dos diversos instrumentos deveria ser melhor investigada, uma vez que os achados do presente estudo não podem ser transcritos para instrumentos maiores ou menores.

Mesmo que o valor encontrado para o peso do instrumento não ultrapasse o recomendado para que o indivíduo possa manter seu alinhamento postural normal (10\% do peso corporal), preocupa-se com a integridade do sistema biológico que pode estar comprometido, pois como afirmam Ren, Jones e Howard ${ }^{21}$ durante a marcha com utilização de sobrecarga é detectada fadiga, sendo que as regiões mais envolvidas são o tronco e os membros inferiores. Quando se afirma que o tronco pode estar comprometido, o efeito destas alterações pode prejudicar o bom desempenho profissional dos músicos. 
A preocupação relacionada ao tema trabalho e acometimento do sistema locomotor crescem com o interesse dos pesquisadores em estudar diversas profissões. Carteiros pedestres durante o transporte em esteira de quatro modelos diferentes de mochilas, também foram estudados. A força vertical apresentou diferenças significativas entre o segundo pico da força vertical entre todas as mochilas. A menor magnitude da força encontrada foi nas mochilas dos tipos alça reta e alça cruzada ${ }^{22}$. Comparando com o presente estudo, a alça reta é a que mais tem semelhança com o tipo de transporte utilizado pelo músico estudado, sendo que, no caso dos carteiros, a mochila era sustentada pelo ombro direito e não pela mão desse membro como no presente estudo.

A curva da força vertical durante a marcha apresenta dois picos como ilustram as Figuras 3 e 4, sendo que o primeiro pico está relacionado à fase de recepção do pé no solo, e o segundo relacionado à fase de propulsão $0^{22}$. Os autores apresentam, geralmente, valores acerca de $1,3 \mathrm{PC}^{23}$ a $1,5 \mathrm{PC}^{24}$ durante os dois picos. A força vertical começa com um pico inicial muito alto, sendo que na fase de apoio total (suporte), esta força cai para um valor inferior do peso corporal, em torno de 0,75 PC e na fase de retirada do pé do solo, os valores novamente ultrapassam o peso corporal. Os valores encontrados para o músico no presente estudo vão ao encontro da literatura pesquisada e são considerados valores baixos $^{23}$.

Assim, como afirmam Peneireiro et al..$^{22}$,as variáveis aqui analisadas estão diretamente relacionadas com sobrecarga mecânica e absorção do impacto. Com isso, existe a necessidade de se averiguar mais profundamente estas relações, dada a influência que estas variáveis podem exercer na condição de saúde daqueles que rotineiramente transportam cargas utilizando mochilas. Durante a realização da marcha o músico apresentou uma maior força vertical sem transportar seu instrumento. Esse fato pode estar relacionado a adaptações no sistema corporal quando se utiliza alguma sobrecarga durante a locomoção. Mais uma vez, estes resultados tornam-se importantes, pois estas diferenças demonstram que mesmo durante um pequeno percurso, como no caso da situação da coleta de dados, o sistema locomotor já apresenta alterações no padrão da marcha, possivelmente, para suprir as necessidades de adaptação muscular que o peso do instrumento obriga o organismo a aderir.

Nota-se que, com a utilização do instrumento, a força vertical máxima além de ser menor demorou também um maior tempo para ocorrer. Estes dados confirmam, mais uma vez, a existência de adaptações do indivíduo durante a realização da marcha com e sem uma sobrecarga. Neste sentido, deve-se notar que a velocidade de execução da marcha pode causar influências, sendo pertinente salientar que durante a caminhada na rua, ao encontro de determinado destino, o sujeito utiliza-se de uma velocidade maior que a utilizada para a coleta de dados e também, em alguns casos, variável. Este fato pode ser ainda mais preocupante uma vez que a velocidade de execução do movimento está correlacionada com os valores da força de reação do solo conforme é abordado por Chung $^{16}$ e Mota e Ribeiro ${ }^{25}$.

O impulso pode ajudar a compreender os fundamentos físicos dos movimentos necessários para o estudo das particularidades específcas das ações motoras, durante a marcha sem e com a utilização do instrumento e está ilustrado na Figura 5. Impulso de uma força se denomina a medida da ação desta força sobre um corpo em um determinado intervalo de tempo.

Neste sentido, valores relativamente mais baixos que os mostrados no presente estudo, foram encontrados no estudo de Wieczorek et al. ${ }^{26}$, que mostram valores referentes ao impulso entre 0,28 PC.s e 0,34 PC.s quando o movimento do step foi analisado em diferentes cadências e alturas. Esses valores apresentam-se inferiores aos encontrados durante a marcha do músico. Obviamente, o step é uma modalidade desenvolvida para fins aeróbicos e o presente estudo buscou avaliar simplesmente a marcha, não como uma atividade que vise aprimoramento do sistema cardiovascular, mas sim, como meio que o individuo utiliza para locomoção. Os valores encontrados para esta variável foram $0,49 \pm 0,18$ PC.s sem a utilização do instrumento e 0,53 $\pm 0,01$ PC.s com a utilização do instrumento. Verificou-se, no caso da variável impulso, maiores valores com o transporte de instrumento, o que pode ser relacionado ao maior peso sustentado pelo indivíduo.

As diferenças encontradas no estudo de Wieczorek et al. ${ }^{26}$ que avaliou a modalidade de step, podem ter ocorrido devido à utilização das cadências e alturas que, por sua vez, 
fazem com que os indivíduos apresentem um tempo de apoio ao solo menor, reduzindo também o impulso. Curiosamente, os valores apresentam-se superiores aos da modalidade de step. A situação 2 mostrou valores maiores, isso demonstra que com a utilização de sobrecarga externa o corpo necessita de um maior impulso para que o movimento ocorra, ou seja, o corpo necessitou de maior energia para realização da atividade e, assim, como afirma o trabalho de Peneireiro et al. ${ }^{22}$, existe a ocorrência de importantes alterações no padrão da marcha em função do modelo de mochila utilizado. Essas variações, já apontadas pela literatura, estão relacionadas com ajustes realizados pelo aparelho locomotor em busca da diminuição do estresse mecânico ${ }^{27}$.

\section{CONCLUSÃO}

As variáveis referentes à força vertical máxima e ao impulso durante a marcha mostraram-se diferentes nas situações sem e com o transporte do instrumento. Isso mostra que ao transportar uma carga, que nesse caso foi de $6,9 \mathrm{~kg}$, o aparelho locomotor sofre alterações, apresentando maiores valores quando o corpo necessita transportar o instrumento de trabalho. Essas alterações, no decorrer do tempo, poderão causar importantes disfunções músculo-esquelético, influenciando na saúde e qualidade de vida do trabalhador se medidas preventivas não forem tomadas.

Sugerem-se novos estudos não só com avaliações cinéticas, mas também que visem avaliar e compreender alterações cinemáticas e eletromiográficas durante o transporte de instrumentos de trabalho, para a certificação da influência deste transporte na saúde e também no desempenho dos trabalhadores diante do estresse aplicado ao organismo.

\section{REFERÊNCIAS BIBLIOGRÁFICAS}

1. Kaneko Y, Lianza S, Dawson WJ. Pain as an incapacitating factor in symphony orchestra musicians in São Paulo, Brazil. Med Probl Perform Art 2005;20(4):168-174.

2. Larsson LG, Baum J, Mudholkar GS, Kollia GD. Nature and impact of musculoskeletal problems in a population of musicians. Med Probl Perform Art 1993;8(3):73-76.

3. Pak CH, Chesky K. Prevalence of hand, finger and wrist musculoskeletal problems in keyboard instrumentalists: the University of North Texas Musician Health Survey. Med Probl Perform Art 2001;16(1):7-23.

4. Tulchinsky E, Riolo L. A biomechanical motion analysis of the violinist's bow arm. Med Probl Perform Art 1994;9(4):119-124.

5. Costa CP, Abrahão JI. Quando o tocar dói: um olhar ergonômico sobre o fazer musical. Per Musi 2004;10:60-79.

6. Costa CP. Contribuições da ergonomia à saúde do músico: considerações sobre a dimensão física do fazer musical. Música Hodie 2005;5(2):53-63.

7. Blum J, Ahlers J. Ergonomic considerations in violist's left shoulder pain. Med Probl Perform Art 1994;9(1):25-29.

8. Winspur I, Wynn Parry CB. The musician's hand. J Hand Surg 1997;22B(4):433-440.

9. Bertoncello D, Menegon NL, Bernardino MM, Camarotto JA. Desenvolvimento conceitual da bolsa do carteiro. Anais eletrônicos do XII Congresso Brasileiro de Ergonomia. Fortaleza: ABERGO; 2004.

10. Cavanagh PRA. Technique for averaging center of pressure paths from a force plataform. J Biomech 1978;11(10-12):478-491.

11. McNair PJ, Prapavessis H. Normative data of vertical ground reaction forces during landing from a jump. J Sci Med Sport 1999;2(1):86-88.

12. Birrell SA, Hooper RH, Haslam RA. The effect of military load carriage on ground reaction forces. Gait Post 2007;26(4):611-614.

13. Larsen AH, Puggaard L, Hämäläinen, U, Aagaard P. Comparison of ground reaction forces and antagonist muscle coactivation during stair walking with ageing. J Electromyogr Kinesiol 2008;18(4):568-580.

14. Winter DA. Knowledge base for diagnostic gait assessments. Med Prog Technol 1993;19(2):61-81

15. Wyss UP, Knuesel O, Gross W. Siple data presentation of gait studies. International Series on Biomechanics. 1987;6:393-398.

16. Chung, TM. Avaliação cinética e cinemática da marcha de adultos do sexo masculino. Acta Fisiatr 2000;7(2):61-67.

17. Finotti RL, Schlee G, Ávila AOV. Efeito do incremento de sobrecarga sobre variáveis cinéticas da marcha em atletas de voleibol. Rev Bras Biomec 2005;6(10):59-63.

18. Hong Y, Li JX. Influence of load and carrying methods on gait phase and ground reactions in children's stair walking. Gait Post 2005;22 (1):63-68.

19. Pardini R, Matsudo S, Araújo T, Matsudo V, Andrade E, Braggion G, Andrade D, Oliveira L, Figueira Júnior A, Raso V. Validação do questionário internacional de nível de atividade física (IPAQ - versão 6): estudo piloto em adultos jovens brasileiros. Rev Bras Ciên Mov 2001;9(3): 45-51.

20. Page BGA. Biomechanical comparison of current 
mailbag designs. Ann Arbor (MI): University of Michigan, 1984.

21. Ren L, Jones R, Howard D. Dynamic analysis of load carriage biomechanics during level walking. J Biomech 2005;38(4):853-863.

22. Peneireiro GM, Amadio AC, Serrão JC. Influência da mochila na característica do padrão dinâmico da locomoção de carteiros pedestres. Anais eletrônicos do XI Congresso Brasileiro de Biomecânica. João Pessoa: UFP; 2005.

23. Amadio AC, Duarte M. Fundamentos biomecânico para a análise do movimento humano. 1st ed. São Paulo (SP): Laboratório de Biomecânica da Escola de Educação Física e Esporte da Universidade de São Paulo, 1996.

24. Carpenter CS. Biomecânica. 1st ed. Rio de Janeiro (RJ): Sprint, 2005.

25. Mota CB, Ribeiro JK. Comportamento da força de reação do solo durante a realização da marcha na ginástica de academia. Rev Bras Biomec 2004;5(8):49-55.
26. Wieczorek AS, Duarte M, Amadio AC. Estudo da força reação do solo no movimento básico de "step". Rev Paul Educ Fís 1997;11(2):103-115.

27. Serrão JC, Sá MR, Amadio AC. Influência dos calçados de futsal no desempenho. Rev Bras Biomec 2000;1(1):39-47. 